\section{Registre français}

\section{Anne Bøggild Bennekou*}

\section{A}

adjectif

antéposé 16 241ff

epithète 16 241-245

postposé 16 241ff

agent-sujet-topique 30170

ambiguïté du sens

ex. de terrible 9 182ff

Anscombre, J.-C.

théorie des topoi $15155 \mathrm{ff}$

argumentation 16 107-114

Aristote

classification aristotélicienne $\mathbf{1 3}$

$213 f$

distinction aristotélicienne $\mathbf{1 3} 214$

article

article générique $13223 f f$

Asher, Nicholas

interprétation du discours $\mathbf{1 6}$ 96ff

Augustin

signification linguistique $\mathbf{4} 10$

B

Benveniste, E.

présent historique 22 81ff

Berthonneau, A.-M.

imparfait comme temps anaphorique méronomique $1698 \mathrm{f}$ interprétation du discours 16 98ff

Blasco-Dulbecco, M.

dislocations en français
Blinkenberg/Høybye

Fondation Blinkenberg/Høybye

1095

Blume, Peter

informations juridiques 4 145-157

Boysen, Gerhard

Blinkenberg/Høybye 10101

C

car 34 169-193

vs. parce que 34190 (fig)

catastrophes, théorie des 9 181-190

Petitot, Jean 9 189f

catégorisation

catégorie de base $\mathbf{1 3} 213$

verticale 13213

causatives 30 167ff

cohésion

cohésion dynamique 78

cohésion statique 78

cohésion textuelle 16 107-114

schéma de cohésion (SDC) 7 7ff

common law

hypercodification 34 243-262

comparaison

en français 19 55-68

comparatif

analytique 19 58ff

synthétique $1958 \mathrm{ff}$

complément

circonstanciel 9 21-22, 22 (fig), 30ff

essentiel 9 21f, 22 (fig), 30ff

partitif 931

compositionnalité, le principe de 4

17-19

connecteur 34182 (fig)

cause vs. raison $34171 \mathrm{ff}$

fonctions pragmatiques 34 170ff

* Anne Bøggild Bennekou

Prs. Charlottes Gade 41, 3. t.v.

DK-2200 København N

annebennekou@hotmail.com

Hermes - Journal of Language and Communication Studies no 39-2007 
fonctions sémantiques 34 170ff motivation 34 177ff

orientation 34182

parce que vs. car 34190 (fig)

Connolly, John $\mathrm{H}$.

grammaire fonctionnelle 22170

craindre 15 172ff

Crevels, Mily

grammaire fonctionnelle 22170

Culler, J.

hypercodification 34247

cusp 9185

\section{D}

Dictionnaire Danois-Francais (B/H) compte-rendu 10 95-102

dictionnaires bilangues différenciation sémantique 8 25-26 information syntaxique 8 26-27

Dik, Simon

coordination 34171

grammaire fonctionnelle 22 168ff passif $30171 \mathrm{ff}$

discours

anaphore 16 93-105

approche lexicaliste 16 100ff

aspect 16 93-105

cohérence de discours 34 169-193

discours juridique 34247

interprétation 16 93-105

marqueurs de discours 34 169-193

marqueurs de cause 34 186ff

dislocations en français 26 165-168

Dobrovie-Sorin, C.

impératif et $3^{\text {e }}$ personne 9176

Dougherty, J.W.D.

dénomination 13215

Ducrot, Oswald

théorie de la polyphonie $\mathbf{1 5} 155 \mathrm{ff}$

théorie des topoi $15155 \mathrm{ff}$

dynamisme sémantique 9 181-190

$\mathbf{E}$
Eco, Umberto

langue et culture $34250 \mathrm{f}$

en plein 16 107-114

énoncé

modalités d'énonciation 9 157ff

relations transphrastiques 78

expansion

circonstancielle 9 21f

essentielle 9 21f

valentielle $923 \mathrm{f}$

$\mathbf{F}$

futur

futur périphrastique 19 279-282

futur simple 19 279-282

G

Gény, F.

constructions juridiques 34 259f

identité juridique 34251

grammaire fonctionnelle 22 168-171, 30160

\section{H}

Helland, H.P.

verbes 19 279-282

Herslund, Michael

Blinkenberg/Høybye 10101

présent historique $2282 \mathrm{ff}$

valence 9 20ff

hypercodification

de la common law 34 243-262

formalisation $34243 f f$

\section{I}

ils générique textuel indirect $\mathbf{1 3}$ 226ff Imbs, P.

présent historique 22 81ff

imparfait

comme temps anaphorique méronomique 16 98f 
impératif

absence de sujet 9162

construction référentielle 9 176ff

emplacement des pronoms 9165

en anglais contemporain 9 157-180

en francais contemporain 9 157-180

groupes nominaux sujet (anglais)

9 172ff

la marque /z/ 9167

négation 9166

première pers. du pluriel 9163

proprietés de l'énoncé impératif $\mathbf{9}$

$161 \mathrm{ff}$

sans sujet apparent (anglais) 9 174f

schémas intonatifs (anglais) 9 170ff

voire/voir 9 168f

implicités

pragmatiques $16262 \mathrm{f}$

sémantiques $16262 \mathrm{f}$

indicatif

vs. subjonctif 15 156ff

information

progression 710 (fig), 17 (fig)

Information legale 4153

$\mathbf{K}$

Kamp, Hans

interprétation du discours 16 94ff

Kleiber, Georges

imparfait comme temps anaphorique méronomique $1698 \mathrm{f}$

interprétation du discours 16 98ff

Klum, A.

présent historique 2286

$\mathbf{L}$

Langacker, R.W.

perception/conception 13217

langage

philosophie 412

Larsson, Björn

adjectifs épithètes 16 241-245

Lascarides, Alex interprétation du discours 16 96ff

Legendre, Pierre

hypercodification 34248

lexème

lexème verbal déclencheur du

subjonctif $15171 \mathrm{f}$

lexicographie

bilangue 8 23ff

équivalents $823 \mathrm{ff}$

microstructure 8 23ff

monolangue 8 23ff

lexie 49

Liedtke, Frank

grammaire fonctionnelle 22169

linguistique modulaire 16 255-264

linguistique textuelle déscription dynamique 1627

diversité des textes 16 16f

langues de specialité 7 7-27

science des textes $\mathbf{1 6} 15 \mathrm{f}$

lix 4 149ff

\section{M}

Mackenzie, John

grammaire fonctionnelle 22171

Martin, R

présent historique 22 81ff

Martinez Caro, Elena

grammaire fonctionnelle 22170

Mellet, S.

présent historique 2288

modi significanti

théorie 411

modifieur 922 (fig)

Molendijk, Arie

interprétation du discours 16 97ff

morphèmes

polysémie 416

référence 4 17ff

sémèmes $\mathbf{4} 16$

sens 4 14f

signification 4 15f

mot

contexte 4 19-27 
notion 4 10ff

Moutaouakil, Ahmed

grammaire fonctionnelle 22169

\section{$\mathbf{N}$}

ne explétif 15 172ff

négation 15 155-176

Nølke, Henning

linguistique modulaire 16255

264

nom d'agent

formation 30161

nominalisation

nucléaire 9 24ff

objective 9 24ff

subjective $924 \mathrm{ff}$

O

onomastique, la thèse 410

$\mathbf{P}$

parce que 34 169-193

vs. car 34190 (fig)

passif $30170 \mathrm{ff}$

changement des fonctions séman-

tiques 30 172ff

phrase

actant fondamental 920 f

catégorisation 13 213-231

circonstant $920 \mathrm{ff}$

hiérarchie 13 213-231

modèle hiérarchique 13217

niveau de base 13214

propriétés communicationnelles

13216

polyphonie 15 155-176

portée, la 16 258, 261 (déf)

pragmatique

temporelle 19 279-282

prédicat

causatif

formation 30162 causatives 30 167ff

forme 30161

prédicat-input 30 159ff

prédicat-output 30 159ff

règles de formation 30 159-175

type $30161 \mathrm{f}$

valence 30 162ff

valence qualitative $30166 f$

présent historique 22 81-97

pronom

pronoms de $3^{\mathrm{e}}$ personne $\mathbf{1 3} 225 \mathrm{f}$

pronoms sans antécédent $13228 \mathrm{ff}$

propositions

appositionnelles 937

concessives $\mathbf{9} 37$

infinitives 937

relatives $936 \mathrm{f}$

puisque 34 169-193

R

Rastier, François

niveau de base 13214

référence 4 9-27

référent

interprétation $13218 f f$

types de référent $13218 f f$

réversibilité

lexicale $1956 f$

morphologique $1956 f$

réversibilité en français 19 55-68

Reyle, Uwe

interprétation du discours 1694

Rivara, René

comparaison 1955

Rohrer, Christian

interprétation du discours 1695

Rosch, E.

modèle psychologique hiérarchique $13213 \mathrm{ff}$

S

sémantique

analyse sémantique (outils) 1631 
formes sémantiques $1626 f$ temporelle 19 279-282

sémantique des textes $\mathbf{1 6}$ 15-36

herméneutique critique 1634

le problème de l'interprétation $\mathbf{1 6}$ 33

l’ordre herméneutique 1635

l’ordre référentiel 1634

sèmes

activation 4 25-26

inhibition 425

propagation 4 26-27

sémiotique

tradition 4 9-13

sens 4 9-27

Serbat, G.

temps 22 91f

Sherry, $\mathrm{S}$.

communication 34 251f

Siewierska, Anna

grammaire fonctionnelle 22170

signification 4 9-27

Soelberg, N.

Blinkenberg/Høybye 10101

Sørensen, Finn

valence $920 \mathrm{ff}$

stabilité structurelle $\mathbf{9} 184$

subjonctif 15 155-176, 156 (déf)

et argumentation 15 156ff

certitude vs. non certitude $\mathbf{1 5}$ 161f

craindre $15172 \mathrm{ff}$

lexème verbal déclencheur du

subjonctif $15171 \mathrm{f}$

ne expletif $15172 \mathrm{ff}$

négation 15 155-176

polyphonie 15 155-176

subordination et polyphonie 15 161f

verbe déclencheur du subjonctif

$15169 f$

substantif

non-prédicatif 938 (fig)

noyau $927 \mathrm{ff}$

prédicatif 921 (déf), 38 (fig)

superlatif

analytique 19 63ff synthétique 19 63ff

syntagmes

participiaux $935 \mathrm{f}$

prépositionnels $934 \mathrm{f}$

pronoms de $3^{\text {e }}$ personne $\mathbf{1 3} 225 \mathrm{f}$

syntagmes nominaux complexes

9 19-41

syntagmes nominaux génériques

13219

Szabolcsi, A

sens litteral 417

\section{$\mathbf{T}$}

TAL (Traitements automatiques du langage) 1633

Télétel 4154

temps

actuel/non-actuel 2284 (fig)

approche aspectuelle 22 91ff

approche du repérage temporel 22

93ff

approche modo-temporelle 22 83ff approches traditionnelles 22 82f

termes de base 13 213-231

terrible

ambiguïte de sens 9 182ff

territoire linguistique 34 247f

Tesnière, Lucien

valence 9 20ff

texte

approches sémantiques $\mathbf{1 6}$ 17ff

formes sémantiques $1626 f$

genres textuels 16 32f

paramètres textuels 30 159-175

l'univers 1625

textes juridiques

exemple de common law 34 245ff

discours juridique 34247

français

syntagmes nominaux

complexes 9 19-41

identité juridique 34 251ff

inconstance des mots 34 259ff

langue du droit 34 249ff 
termes diviseurs (en anglais) 34

$254 \mathrm{ff}$

territoire linguistique $34247 \mathrm{f}$

types d'abréviations 34 255ff

thème

thème générique 1622 (déf)

thème spécifique 1623 (déf)

thème-rhème 7 13-16

théories du texte

linguistiques $1617 f$

sémiotiques $1617 \mathrm{f}$

typologie du texte

quatre composantes $1621 \mathrm{ff}$

niveau agonistique 16 24f

V

Van den Berg, Marinus

grammaire fonctionnelle 22169

verbe

déclencheur du subjonctif 15 169f

interaction verbale 22 168-171

Vet, Co

grammaire fonctionnelle 22169

W

Weinrich, $\mathrm{H}$.

présent historique 22 81ff

Wierzbicka, A.

classification 13 214ff

Wilmet, $\mathrm{M}$.

présent historique 22 81ff 